\title{
Bipolar Fuzzy Graphs with Categorical Properties
}

\author{
Hossein Rashmanlou $^{1}$, Sovan Samanta ${ }^{2}$, Madhumangal Pal ${ }^{3}$, R.A.Borzooei ${ }^{1}$ \\ ${ }^{1}$ Department of Mathematics, Islamic Azad University, Central Tehran Branch, \\ Tehran, Iran \\ E-mail: rashmanlou.1987@gmail.com,borzooei@sbu.ac.ir \\ ${ }^{2}$ Department of Mathematics, Joykrishnapur High School (H.S.), \\ Simulia, Tamluk, \\ West Bengal-721649, India \\ E-mail: ssamantavu@gmail.com \\ ${ }^{3}$ Department of Applied Mathematics, Vidyasagar University, \\ Midnapore-721102, India \\ E-mail:mmpalvu@gmail.com
}

Received 29 April 2014

Accepted 11 May 2015

\begin{abstract}
Theoretical concepts of graphs are highly utilized by computer science applications. Especially in research areas of computer science such as data mining, image segmentation, clustering, image capturing and networking. In this paper, we discussed some properties of the $\mu$-complement of bipolar fuzzy graphs. Self $\mu$-complement bipolar fuzzy graphs and self weak $\mu$-complement bipolar fuzzy graphs are defined and a necessary condition for a bipolar fuzzy graph to be self $\mu$-complement is given. We defined busy vertices and free vertices in bipolar fuzzy graphs and studied their image under an isomorphism. Categorical properties of bipolar fuzzy graphs are discussed. Also, we investigated some properties of isomorphism on bipolar fuzzy graphs.
\end{abstract}

Keywords: Bipolar fuzzy graphs, $\mu$-complement and self $\mu$-complement, Busy vertex and Free vertex.

\section{Introduction}

Presently, science and technology is featured with complex processes and phenomena for which complete information is not always available. For such cases, mathematical models are developed to handle various types of systems containing elements of uncertainty. A large number of these models is based on an extension of the ordinary set theory, namely, fuzzy sets. Graph theory has numerous applications to problem in computer science, electrical engineering, system analysis, operations re- search, economics, networking routing, transportation, etc. In 1965, Zadeh [28] introduced the notion of a fuzzy subset of a set as a method for representing uncertainty. In 1975, Rosenfeld [11] introduced the notion of fuzzy graphs and proposed another definitions including paths, cycles, connectedness, etc. The complement of a fuzzy graph was defined by Mordeson and Nair [9] and further studied by Sunitha and Kumar [14]. The concept of weak isomorphism, co-weak isomorphism and isomorphism between fuzzy graphs was introduced by Bhutani 
in [3]. Nagoorgani and Chandrasekaran [10] defined $\mu$-complement of a fuzzy graph. After that, Samanta and Pal introduced several types of fuzzy graphs like fuzzy planar graphs [15], fuzzy competition graph [17, 22], fuzzy tolerance graphs [16], fuzzy threshold graphs [18].

In 1994, Zhang [31,32] initiated the concept of bipolar fuzzy sets as a generalization of fuzzy sets. Bipolar fuzzy set is an extension of fuzzy set. In this set, there are two types of membership values one lies in $[-1,0]$ and other in $[0,1]$, called negative and positive membership values. The first definition of bipolar fuzzy graphs was proposed by Akram [1]. In 2011, Akram and Dudek [2] defined regular bipolar fuzzy graphs and introduced the concept of regular and totally regular bipolar fuzzy graphs. After that, several researches are doing on these graphs. Samanta and Pal discussed some properties of bipolar fuzzy graphs in [19-21].

In this paper, we defined $\mu$-complement of bipolar fuzzy graphs and investigated some properties of it. Busy vertices and free vertices in bipolar fuzzy graphs are defined. Categorical properties of bipolar fuzzy graphs are discussed. Lastly, some properties of isomorphism are studied on bipolar fuzzy graphs. For other notations, terminologies and applications not mentioned in the paper, the readers are referred to $[23-25,29,30,32,33]$.

\section{Preliminaries}

The main objective of this paper is to study of bipolar fuzzy graph and this graph is based on the bipolar fuzzy set defined below.

Let $X$ be a non-empty set. A bipolar fuzzy set $B$ in $X$ is an object having the form $B=$ $\left\{\left(x, \mu_{B}^{P}(x), \mu_{B}^{N}(x)\right) \mid x \in X\right\}$, where $\mu_{B}^{P}: X \rightarrow[0,1]$ and $\mu_{B}^{N}: X \rightarrow[-1,0]$ are mappings. We use the positive membership degree $\mu_{B}^{P}(x)$ to denote the satisfaction degree of an element $x$ to the property corresponding to a bipolar fuzzy set $B$, and the negative membership degree $\mu_{B}^{N}(x)$ to denote the satisfaction degree of an element $x$ to some implicit counterproperty corresponding to a bipolar fuzzy set $B$. If $\mu_{B}^{P}(x) \neq 0$ and $\mu_{B}^{N}(x)=0$, it is the situation that $x$ is regarded as having only positive satisfaction for $B$.
If $\mu_{B}^{P}(x)=0$ and $\mu_{B}^{N}(x) \neq 0$, it is the situation that $x$ does not satisfy the property of $B$ but somewhat satisfies the counter property of $B$. It is possible for an element $x$ to be such that $\mu_{B}^{P}(x) \neq 0$ and $\mu_{B}^{N}(x) \neq 0$ when the membership function of the property overlaps that of its counter property over some portion of $X$.

For the sake of simplicity, we shall use the symbol $B=\left(\mu_{B}^{P}, \mu_{B}^{N}\right)$ for the bipolar fuzzy set $B$ $=\left\{\left(x, \mu_{B}^{P}(x), \mu_{B}^{N}(x)\right) \mid x \in X\right\}$.

Let $X$ be a non-empty set. Then we call a map$\operatorname{ping} A=\left(\mu_{A}^{P}, \mu_{A}^{N}\right): X \times X \rightarrow[0,1] \times[-1,0]$ a bipolar fuzzy relation on $X$ such that $\mu_{A}^{P}(x, y) \in[0,1]$ and $\mu_{A}^{N}(x, y) \in[-1,0]$.

Let $A=\left(\mu_{A}^{P}, \mu_{A}^{N}\right)$ and $B=\left(\mu_{B}^{P}, \mu_{B}^{N}\right)$ be two bipolar fuzzy sets on a set $X$. If $A=\left(\mu_{A}^{P}, \mu_{A}^{N}\right)$ is a bipolar fuzzy relation on a set $X$, then $A=\left(\mu_{A}^{P}, \mu_{A}^{N}\right)$ is called a bipolar fuzzy relation on $B=\left(\mu_{B}^{P}, \mu_{B}^{N}\right)$ if $\mu_{A}^{P}(x, y) \leqslant \min \left(\mu_{B}^{P}(x), \mu_{B}^{P}(y)\right)$ and $\mu_{A}^{N}(x, y) \geqslant$ $\max \left(\mu_{B}^{N}(x), \mu_{B}^{N}(y)\right)$ for all $x, y \in X$. A bipolar fuzzy relation $A$ on $X$ is called symmetric if $\mu_{A}^{P}(x, y)$ $=\mu_{A}^{P}(y, x)$ and $\mu_{A}^{N}(x, y)=\mu_{A}^{N}(y, x)$ for all $x, y \in X$.

Definition 1. Let $V$ be a nonempty set. A bipolar fuzzy graph is a triple $G=(V, A, B)$, where $A=\left(\mu_{A}^{P}, \mu_{A}^{N}\right)$ is a bipolar fuzzy set on $V$ and $B$ $=\left(\mu_{B}^{P}, \mu_{B}^{N}\right)$ is a bipolar fuzzy relation on $E \subset V \times V$ such that

$\mu_{B}^{P}(x y) \leqslant \min \left(\mu_{A}^{P}(x), \mu_{A}^{P}(y)\right)$ and $\mu_{B}^{N}(x y) \geqslant$ $\max \left(\mu_{A}^{N}(x), \mu_{A}^{N}(y)\right)$ for all $x y \in E, V$ is called the set of vertices and $E$ is called the set of edges.

Definition 2. Let $G_{1}=\left(V_{1}, A_{1}, B_{1}\right)$ and $G_{2}=$ $\left(V_{2}, A_{2}, B_{2}\right)$ be the bipolar fuzzy graphs. A homomorphism $f$ from $G_{1}$ to $G_{2}$ is a mapping $f: V_{1} \rightarrow V_{2}$ which satisfies the following conditions:

(a) $\mu_{A_{1}}^{P}\left(x_{1}\right) \leqslant \mu_{A_{2}}^{P}\left(f\left(x_{1}\right)\right), \mu_{A_{1}}^{N}\left(x_{1}\right) \geqslant \mu_{A_{2}}^{N}\left(f\left(x_{1}\right)\right)$,

(b) $\mu_{B_{1}}^{P}\left(x_{1} y_{1}\right) \leqslant \mu_{B_{2}}^{P}\left(f\left(x_{1}\right) f\left(y_{1}\right)\right), \mu_{B_{1}}^{N}\left(x_{1} y_{1}\right) \geqslant$ $\mu_{B_{2}}^{N}\left(f\left(x_{1}\right) f\left(y_{1}\right)\right)$ for all $x_{1}, y_{1} \in V_{1}, x_{1} y_{1} \in E_{1}$.

Definition 3. Let $G_{1}$ and $G_{2}$ be bipolar fuzzy graphs. An isomorphism $f$ from $G_{1}$ to $G_{2}$ is a bijective mapping $f: V_{1} \rightarrow V_{2}$ which satisfies the following conditions:

(c) $\mu_{A_{1}}^{P}\left(x_{1}\right)=\mu_{A_{2}}^{P}\left(f\left(x_{1}\right)\right), \mu_{A_{1}}^{N}\left(x_{1}\right)=\mu_{A_{2}}^{N}\left(f\left(x_{1}\right)\right)$, (d) $\mu_{B_{1}}^{P}\left(x_{1} y_{1}\right)=\mu_{B_{2}}^{P}\left(f\left(x_{1}\right) f\left(y_{1}\right)\right), \mu_{B_{1}}^{N}\left(x_{1} y_{1}\right)=\mu_{B_{2}}^{N}$ $\left(f\left(x_{1}\right) f\left(y_{1}\right)\right)$ for all $x_{1}, y_{1} \in V_{1}, x_{1} y_{1} \in E_{1}$. 
Definition 4. Let $G_{1}$ and $G_{2}$ be bipolar fuzzy graphs. Then, a weak isomorphism $f$ from $G_{1}$ to $G_{2}$ is a bijective mapping $f: V_{1} \rightarrow V_{2}$ which satisfies the following conditions:

(e) $f$ is homomorphism

(f) $\mu_{A_{1}}^{P}\left(x_{1}\right)=\mu_{A_{2}}^{P}\left(f\left(x_{1}\right)\right), \mu_{A_{1}}^{N}\left(x_{1}\right)=\mu_{A_{2}}^{N}\left(f\left(x_{1}\right)\right)$, for all $x_{1} \in V_{1}$. Thus a weak isomorphism preserves the membership values for the nodes but not necessarily for the arcs.

Definition 5. Let $G_{1}$ and $G_{2}$ be the bipolar fuzzy graphs. A co-weak isomorphism

$f$ from $G_{1}$ to $G_{2}$ is a bijective mapping $f: V_{1} \rightarrow V_{2}$ which satisfies

$(g) f$ is homomorphism

(h) $\mu_{B_{1}}^{P}\left(x_{1} y_{1}\right)=\mu_{B_{2}}^{P}\left(f\left(x_{1}\right) f\left(y_{1}\right)\right), \mu_{B_{1}}^{N}\left(x_{1} y_{1}\right)=$ $\mu_{B_{2}}^{N}\left(f\left(x_{1} f\left(y_{1}\right)\right)\right.$, for all $x_{1}, y_{1} \in V_{1}$. Thus a co-weak isomorphism preserves the membership values for the arcs but not necessarily for the nodes.

The order and size of a graph represent its dimension. In different graph operations they are generally changed. Unlike crisp graphs, the order and size of a fuzzy graph are different. The definition of order and size of a bipolar fuzzy graph are given below.

Definition 6. The order of a bipolar fuzzy graph $G=(V, A, B)$ is denoted by $|V|$ (or $O(G)$ ), and is defined by

$$
O(G)=|V|=\sum_{x \in V} \frac{1+\mu_{A}^{P}(x)+\mu_{A}^{N}(x)}{2} .
$$

The size of a bipolar fuzzy graph $G=(V, A, B)$ is denoted by $|E|$ (or $S(G)$ ), and is defined by

$$
S(G)=|E|=\sum_{x y \in E} \frac{1+\mu_{B}^{P}(x y)+\mu_{B}^{N}(x y)}{2} .
$$

Definition 7. Let $G$ be a bipolar fuzzy graph. The neighborhood of vertex $x$ in $G$ is defined by $N(x)=N_{P}(x) \cup N_{N}(x)$, where

$N_{P}(x)=\left\{y \in V: \mu_{B^{P}}(x y) \leqslant \min \left(\mu_{A^{P}}(x), \mu_{A^{P}}(y)\right)\right\}$ and $N_{N}(x)=\left\{y \in V: \mu_{B^{N}}(x y) \geqslant \max \left(\mu_{A^{N}}(x), \mu_{A^{N}}(y)\right)\right\}$.

Definition 8. Let $G=(V, A, B)$ be a bipolar fuzzy graph. The neighborhood degree of a vertex $x$ is defined as $\operatorname{deg}(x)=\left(\operatorname{deg}_{P}(x), \operatorname{deg}_{N}(x)\right)$, where $\operatorname{deg}_{P}(x)$ $=\sum_{y \in N(x)} \mu_{A}^{P}(y)$ and $\operatorname{deg}_{N}(x)=\sum_{y \in N(x)} \mu_{A}^{N}(y)$. Notice that $\mu_{B}^{P}(x y)>0, \mu_{B}^{N}(x y)<0$ for $x y \in E$, and $\mu_{B}^{P}(x y)=\mu_{B}^{N}(x y)=0$ for $x y \notin E$.

Definition 9. A path in a bipolar fuzzy graph is a sequence of distinct vertices $v_{1}, v_{2}, \cdots, v_{n+1}$ such that 1. $\mu_{B}^{P}\left(v_{i-1} v_{i}\right)>0$ and $\mu_{B}^{N}\left(v_{i-1} v_{i}\right)=0$ for some vertices $v_{i-1}, v_{i}$.

2. $\mu_{B}^{P}\left(v_{i-1} v_{i}\right)=0$ and $\mu_{B}^{N}\left(v_{i-1} v_{i}\right)<0$ for some vertices $v_{i-1}, v_{i}$.

3. $\mu_{B}^{P}\left(v_{i-1} v_{i}\right)>0$ and $\mu_{B}^{N}\left(v_{i-1} v_{i}\right)<0$.

A path $\rho: v_{1} v_{2} \cdots v_{n+1}$ in $G$ is called a cycle if $v_{1}=v_{n+1}$ and $n \geqslant 3$.

The length of this path is $n$.

Definition 10. In a bipolar fuzzy graph $G$ we have $\mu_{B^{P}}^{K}(u v)=\sup \left\{\mu_{B^{P}}\left(u v_{1}\right) \wedge \mu_{B^{P}}\left(v_{1} v_{2}\right) \wedge \mu_{B^{P}}\left(v_{2} v_{3}\right)\right.$, $\left.\cdots, \wedge \mu_{B^{P}}\left(v_{k-1} v\right) \mid u, v_{1}, v_{2}, \cdots, v_{k-1}, v \in V\right\}$ $\mu_{B^{N}}^{K}(u v)=\sup \left\{\mu_{B^{N}}\left(u v_{1}\right) \wedge \mu_{B^{N}}\left(v_{1} v_{2}\right) \wedge \mu_{B^{N}}\left(v_{2} v_{3}\right)\right.$, $\left.\cdots, \wedge \mu_{B^{P}}\left(v_{k-1} v\right) \mid u, v_{1}, v_{2}, \cdots, v_{k-1}, v \in V\right\}$.

Also we have

$\mu_{B^{P}}^{\infty}(u v)=\sup \left\{\mu_{B^{P}}^{K}(u v) \mid k=1,2,3, \cdots\right\}$ and $\mu_{B^{N}}^{\infty}(u v)=\sup \left\{\mu_{B^{N}}^{K}(u v) \mid k=1,2,3, \cdots\right\}$.

Definition 11. In a bipolar fuzzy graph $G=$ $(V, A, B)$, an arc $(u, v)$ is said to be a strong arc, if $\mu_{B^{P}}(u v) \geqslant \mu_{B^{P}}^{\infty}(u v)$ and $\mu_{B^{N}}(u v) \geqslant \mu_{B^{N}}^{\infty}(u v)$.

\section{3. $\mu$-complement and self $\mu$-complement bipolar fuzzy graphs}

In this section, $\mu$-complement of a bipolar fuzzy graph $G$ is introduced. The $\mu$-complement of a bipolar fuzzy graph $G=(V, A, B)$ is denoted by $G^{\mu}=\left(V, A^{\mu}, B^{\mu}\right)$, which is defined below.

Definition 12. Let $G=(V, A, B)$ be a bipolar fuzzy graph. The $\mu$-complement of $G$ is denoted by $G^{\mu}=\left(V, A^{\mu}, B^{\mu}\right)$, where $A^{\mu}=A, B^{\mu}=\left(\mu_{B^{P}}^{\mu}, \mu_{B^{N}}^{\mu}\right)$ and

$$
\begin{array}{r}
\mu_{B^{P}}^{\mu}(x y)= \begin{cases}\mu_{A}^{P}(x) \wedge \mu_{A}^{P}(y)-\mu_{B^{P}}(x y) & \text { if } \mu_{B^{P}}(x y)>0 \\
0 & \text { if } \mu_{B^{P}}(x y)=0,\end{cases} \\
\mu_{B^{N}}^{\mu}(x y)= \begin{cases}\mu_{A}^{N}(x) \vee \mu_{A}^{N}(y)-\mu_{B^{N}}(x y) & \text { if } \mu_{B^{N}}(x y)<0 \\
0 & \text { if } \mu_{B^{N}}(x y)=0 .\end{cases}
\end{array}
$$

Several properties have been investigated for this graph. 
Proposition 1. Let $G_{1}$ and $G_{2}$ be bipolar fuzzy graphs. If $G_{1}$ and $G_{2}$ are isomorphic, then their $\mu$-complements, $G_{1}^{\mu}$ and $G_{2}^{\mu}$, are also isomorphic.

Proof. Let $G_{1} \cong G_{2}$, and $f$ be an isomorphism from $G_{1}$ to $G_{2}$. Then, $\mu_{A_{1}^{P}}(x)=\mu_{A_{2}^{P}}$ $(f(x)), \mu_{A_{1}^{N}}(x)=\mu_{A_{2}^{N}}(f(x))$ for all $x \in V_{1}, \mu_{B_{1}^{P}}(x y)$ $=\mu_{B_{2}^{P}}(f(x) f(y)), \mu_{B_{1}^{N}}(x y)=\mu_{B_{2}^{N}}(f(x) f(y))$ for all $x y \in E_{1}$. If $\mu_{B_{1}^{P}}(x y)>0$, then $\mu_{B_{2}^{P}}(f(x) f(y))>$ $0, \quad$ and $\mu_{B_{1}^{P}}^{\mu}(x y)=\mu_{A_{1}^{P}}(x) \wedge \mu_{A_{1}^{P}}(y)-\mu_{B_{1}^{P}}(x y)$ $=\mu_{A_{2}^{P}}(f(x)) \wedge \mu_{A_{2}^{P}}(f(y))-\mu_{B_{2}^{P}}(f(x) f(y))=$ $\mu_{B_{2}^{p}}^{\mu}(f(x) f(y))$.

If $\mu_{B_{1}}^{P}(x y)=0$, then $\mu_{B_{2}^{P}}(f(x) f(y))=0$, and $\mu_{B_{1}^{P}}^{\mu}(x y)$ $=0=\mu_{B_{2}^{P}}^{\mu}(f(x) f(y))$.

Thus $\mu_{B_{1}^{p}}^{\mu}(x y)=\mu_{B_{2}^{P}}^{\mu}(f(x) f(y))$ for all $x y \in E_{1}$. Similarly, we can prove that

$\mu_{B_{1}^{N}}^{\mu}(x y)=\mu_{B_{2}^{N}}^{\mu}(f(x) f(y))$ for all $x y \in E_{1}$. Therefore, $f$ from $G_{1}^{\mu}$ to $G_{2}^{\mu}$ is an isomorphism, i.e. $G_{1}^{\mu} \cong G_{2}^{\mu}$.

Theorem 2. Let $G_{1}=\left(V_{1}, A_{1}, B_{1}\right)$ and $G_{2}=$ $\left(V_{2}, A_{2}, B_{2}\right)$ be two bipolar fuzzy graphs. Then, $G_{1}^{\mu}=G_{2}^{\mu}$ if and only if every arc $(x, y)$ satisfying one of the following conditions. (1) $\mu_{B_{1}^{p}}(x y)=$ $\mu_{B_{2}^{P}}(x y), \quad \mu_{B_{1}^{N}}(x y)=\mu_{B_{2}^{N}}(x y)$, (2) $\mu_{B_{1}^{P}}(x y)=$ $\mu_{B_{2}^{P}}(x y), \mu_{B_{2}^{N}}(x y)=0, \mu_{B_{2}^{N}}(x y)=\mu_{A^{N}}(x) \vee \mu_{A^{N}}(y)$, (3) $\quad \mu_{B_{1}^{P}}(x y)=\mu_{B_{2}^{P}}(x y), \quad \mu_{B_{1}^{N}}(x y)=\mu_{A^{N}}(x) \vee$ $\mu_{A^{N}}(y), \mu_{B_{2}^{N}}(x y)=0$,

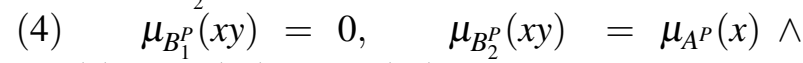
$\mu_{A^{P}}(y), \mu_{B_{1}^{N}}(x y)=\mu_{B_{2}^{N}}(x y)$,

(5) $\quad \mu_{B_{1}^{P}}(x y)=0, \quad \mu_{B_{2}^{P}}(x y)=\mu_{A^{P}}(x) \wedge$ $\mu_{A^{P}}(y), \quad \mu_{B_{1}^{N}}(x y)=0, \quad \mu_{B_{2}^{N}}(x y)^{2}=\mu_{A^{N}}(x) \vee \mu_{A^{N}}(y)$, (6) $\quad \mu_{B_{1}^{P}}(x y)=\mu_{A^{P}}(x) \wedge \mu_{A^{P}}(y), \quad \mu_{B_{2}^{P}}(x y)=$ $0, \mu_{B_{1}^{N}}(x y)=\mu_{B_{2}^{N}}(x y)$,

(7) $\quad \mu_{B_{1}^{P}}(x y)=\mu_{A^{P}}(x) \wedge \mu_{A^{p}}(y), \quad \mu_{B_{2}^{p}}(x y)=$ $0, \mu_{B_{1}^{N}}(x y)=\mu_{A^{N}}(x) \vee \mu_{A^{N}}(y), \mu_{B_{2}^{N}}(x y)=0$.

Theorem 3. Let $G_{1}=\left(V_{1}, A_{1}, B_{1}\right)$ and $G_{2}=$ $\left(V_{2}, A_{2}, B_{2}\right)$ be two bipolar fuzzy graphs such that $V_{1} \cap V_{2}=\phi$. Then, $\left(G_{1}+G_{2}\right)^{\mu} \cong G_{1}^{\mu} \cup G_{2}^{\mu}$.

Proof. Let $I: V_{1} \cup V_{2} \mapsto V_{1} \cup V_{2}$ be the identity map. We prove that for all $x, y \in V$
$\left(\mu_{A_{1}^{P}}+\mu_{A_{2}^{P}}\right)^{\mu}(x)=\mu_{A_{1}^{P}}^{\mu}(x) \cup \mu_{A_{2}^{P}}^{\mu}(x), \quad\left(\mu_{A_{1}^{N}}+\right.$ $\left.\mu_{A_{2}^{N}}\right)^{\mu}(x)=\mu_{A_{1}^{N}}^{\mu}(x) \cup \mu_{A_{2}^{N}}^{\mu}(x)$ and $\left(\mu_{B_{1}^{P}}+\mu_{B_{2}^{P}}\right)^{\mu}(x y)$ $=\mu_{B_{1}^{P}}^{\mu} \cup \mu_{B_{2}^{P}}^{\mu}(x y), \quad\left(\mu_{B_{1}^{N}}+\mu_{B_{2}^{N}}\right)^{\mu}(x y)=\mu_{B_{1}^{N}}^{\mu} \cup$ $\mu_{B_{2}^{N}}^{\mu}(x y)$.

For all $x, y \in V$ we have

$$
\begin{aligned}
\left(\mu_{A_{1}^{P}}+\mu_{A_{2}^{P}}\right)^{\mu}(x) & = \begin{cases}\mu_{A_{1}^{P}}(x) & \text { if } x \in V_{1} \\
\mu_{A_{2}^{P}}(x) & \text { if } x \in V_{2}\end{cases} \\
& = \begin{cases}\mu_{A^{P}}^{\mu}(x) & \text { if } x \in V_{1} \\
\mu_{A_{1}^{N}}^{\mu}(x) & \text { if } x \in V_{2}\end{cases} \\
& =\left(\mu_{A_{1}^{p}}^{\mu} \cup \mu_{A_{2}^{p}}^{\mu}\right)(x), \\
\left(\mu_{A_{1}^{N}}+\mu_{A_{2}^{N}}\right)^{\mu}(x) & = \begin{cases}\mu_{A_{1}^{N}}(x) & \text { if } x \in V_{1} \\
\mu_{A_{2}^{N}}(x) & \text { if } x \in V_{2}\end{cases} \\
& = \begin{cases}\mu_{A_{1}^{N}}^{\mu}(x) & \text { if } x \in V_{1} \\
\mu_{A_{1}^{N}}^{\mu}(x) & \text { if } x \in V_{2}\end{cases} \\
& =\left(\mu_{A_{1}^{N}}^{\mu} \cup \mu_{A_{2}^{N}}^{\mu}\right)(x) .
\end{aligned}
$$

Moreover,

$\left(\mu_{B_{1}^{P}}+\mu_{B_{2}^{P}}\right)^{\mu}(x y)=\left(\mu_{A_{1}^{P}}+\mu_{A_{2}^{P}}\right)(x) \wedge\left(\mu_{A_{1}^{P}}+\right.$ $\left.\mu_{A_{2}^{P}}\right)(y)-\left(\mu_{B_{1}^{P}}+\mu_{B_{2}^{P}}\right)(x y)$

$$
\begin{aligned}
& = \begin{cases}\mu_{A_{1}^{P}}(x) \wedge \mu_{A_{1}^{P}}(y)-\mu_{B_{1}^{P}}(x y) & \text { if } x y \in E_{1} \\
\mu_{A_{2}^{P}}(x) \wedge \mu_{A_{2}^{P}}(y)-\mu_{B_{2}^{P}}(x y) & \text { if } x y \in E_{2} \\
\mu_{A_{1}^{P}}(x) \wedge \mu_{A_{2}^{P}}(y)-\mu_{A_{1}^{P}}(x) \wedge \mu_{A_{2}^{P}}(y) & \text { if } x y \in E^{\prime}\end{cases} \\
& = \begin{cases}\mu_{B_{1}^{p}}^{\mu}(x y) & \text { if } x y \in E_{1} \\
\mu_{B_{2}^{p}}^{\mu}(x y) & \text { if } x y \in E_{2} \\
0 & \text { if } x y \in E^{\prime}\end{cases} \\
& =\left(\mu_{B_{1}^{P}}^{\mu} \cup \mu_{B_{2}^{P}}^{\mu}\right)(x y) \text {, } \\
& \left(\mu_{B_{1}^{N}}+\mu_{B_{2}^{N}}\right)^{\mu}(x y)=\left(\mu_{A_{1}^{N}}+\mu_{A_{2}^{N}}\right)(x) \quad \vee\left(\mu_{A_{1}^{N}}+\right. \\
& \left.\mu_{A_{2}^{N}}\right)(y)-\left(\mu_{B_{1}^{N}}+\mu_{B_{2}^{N}}\right)(x y) \\
& = \begin{cases}\mu_{A_{1}^{N}}(x) \vee \mu_{A_{1}^{N}}(y)-\mu_{B_{1}^{N}}(x y) & \text { if } x y \in E_{1} \\
\mu_{A_{2}^{N}}(x) \vee \mu_{A_{2}^{N}}(y)-\mu_{B_{2}^{N}}(x y) & \text { if } x y \in E_{2} \\
\mu_{A_{1}^{N}}(x) \vee \mu_{A_{1}^{N}}(y)-\mu_{A_{1}^{N}}(x) \vee \mu_{A_{1}^{N}}(y) & \text { if } x y \in E^{\prime}\end{cases} \\
& = \begin{cases}\mu_{B_{1}^{N}}^{\mu}(x y) & \text { if } x y \in E_{1} \\
\mu_{B_{2}^{N}}^{\mu}(x y) & \text { if } x y \in E_{2} \\
0 & \text { if } x y \in E^{\prime}\end{cases} \\
& =\left(\mu_{B_{1}^{N}}^{\mu} \cup \mu_{B_{2}^{N}}^{\mu}\right)(x y) \text {. }
\end{aligned}
$$


Theorem 4. Let $G_{1}=\left(V_{1}, A_{1}, B_{1}\right)$ and $G_{2}=$ $\left(V_{2}, A_{2}, B_{2}\right)$ be two bipolar fuzzy graphs such that $V_{1} \cap V_{2}=\phi$. Then, $\left(G_{1} \cup G_{2}\right)^{\mu} \cong G_{1}^{\mu} \cup G_{2}^{\mu}$.

Proof. We shall prove that the identity map is the required isomorphism. If $x \in V_{1}$

$\left(\mu_{A_{1}^{P}} \cup \mu_{A_{2}^{P}}\right)^{\mu}(x)=\left(\mu_{A_{1}^{P}} \cup \mu_{A_{2}^{P}}\right)(x)=\mu_{A_{1}^{P}}(x)=\mu_{A_{1}^{P}}^{\mu}$ $=\left(\mu_{A_{1}^{P}}^{\mu} \cup \mu_{A_{2}^{P}}^{\mu}\right)(x)$,

$\left(\mu_{A_{1}^{N}} \cup \mu_{A_{2}^{N}}\right)^{\mu}(x)=\left(\mu_{A_{1}^{N}} \cup \mu_{A_{2}^{N}}\right)(x)=\mu_{A_{1}^{N}}(x)=\mu_{A_{1}^{N}}^{\mu}$ $=\left(\mu_{A_{1}^{N}}^{\mu} \cup \mu_{A_{2}^{N}}^{\mu}\right)(x)$.

If $x \in V_{2}$, then

$\left(\mu_{A_{1}^{P}} \cup \mu_{A_{2}^{P}}\right)^{\mu}(x)=\left(\mu_{A_{1}^{P}} \cup \mu_{A_{2}^{P}}\right)(x)=\mu_{A_{2}^{P}}(x)=\mu_{A_{2}^{P}}^{\mu}$ $=\left(\mu_{A_{1}^{P}}^{\mu} \cup \mu_{A_{2}^{P}}^{\mu}\right)(x)$,

$\left(\mu_{A_{1}^{N}} \cup \mu_{A_{2}^{N}}\right)^{\mu}(x)=\left(\mu_{A_{1}^{N}} \cup \mu_{A_{2}^{N}}\right)(x)=\mu_{A_{2}^{N}}(x)=\mu_{A_{2}^{N}}^{\mu}$ $=\left(\mu_{A_{1}^{N}}^{\mu} \cup \mu_{A_{2}^{N}}^{\mu}\right)(x)$.

If $x y \in E_{1}$, then

$$
\begin{aligned}
\left(\mu_{B_{1}^{P}} \cup \mu_{B_{2}^{P}}\right)^{\mu}(x y) & =\mu_{A_{1}^{P}}(x) \wedge \mu_{A_{1}^{P}}(y)-\mu_{B_{1}^{P}}(x y) \\
& =\mu_{B_{1}^{P}}^{\mu}(x y)=\left(\mu_{B_{1}^{p}}^{\mu} \cup \mu_{B_{2}^{p}}^{\mu}(x y)\right. \\
& =\left(\mu_{B_{1}^{P}}^{\mu} \cup \mu_{B_{2}^{p}}^{\mu}\right)(x y) . \\
\left(\mu_{B_{1}^{N}} \cup \mu_{B_{2}^{N}}\right)^{\mu}(x y) & =\mu_{A_{1}^{N}}(x) \vee \mu_{A_{1}^{N}}(y)-\mu_{B_{1}^{N}}(x y) \\
& =\mu_{B_{1}^{N}}^{\mu}(x y)=\left(\mu_{B_{1}^{N}}^{\mu} \cup \mu_{B_{2}^{N}}^{\mu}\right)(x y) \\
& =\left(\mu_{B_{1}^{N}}^{\mu} \cup \mu_{B_{2}^{N}}^{\mu}\right)(x y) .
\end{aligned}
$$

If $x y \in E_{2}$, then

$$
\begin{aligned}
\left(\mu_{B_{1}^{p}} \cup \mu_{B_{2}^{P}}\right)^{\mu}(x y) & =\mu_{A_{2}^{P}}(x) \wedge \mu_{A_{2}^{P}}(y)-\mu_{B_{2}^{P}}(x y) \\
& =\mu_{B_{2}^{P}}^{\mu}(x y)=\left(\mu_{B_{1}^{p}}^{\mu} \cup \mu_{B_{2}^{p}}^{\mu}(x y)\right. \\
& =\left(\mu_{B_{1}^{p}}^{\mu} \cup \mu_{B_{2}^{p}}^{\mu}(x y)\right. \\
\left(\mu_{B_{1}^{N}} \cup \mu_{B_{2}^{N}}\right)^{\mu}(x y) & =\mu_{A_{2}^{N}}(x) \vee \mu_{A_{2}^{N}}(y)-\mu_{B_{2}^{N}}(x y) \\
& =\mu_{B_{2}^{N}}^{\mu}(x y)=\left(\mu_{B_{1}^{N}}^{\mu} \cup \mu_{B_{2}^{N}}^{\mu}\right)(x y) \\
& =\left(\mu_{B_{1}^{N}}^{\mu} \cup \mu_{B_{2}^{N}}^{\mu}\right)(x y) .
\end{aligned}
$$

\section{Busy vertices and free vertices in bipolar fuzzy graphs}

In this section, we defined two special types of vertices called busy and free vertices of a bipolar fuzzy graphs.
Definition 13. The busy value of a node $v$ of a bipolar fuzzy graph $G=(V, A, B)$ is defined to be $D(v)=$ $\left(D_{P}(v), D_{N}(v)\right)$ where $D_{P}(v)=\sum_{i} \mu_{A^{P}}(v) \wedge \mu_{A^{P}}\left(v_{i}\right)$ and $D_{N}(v)=\sum_{i} \mu_{A^{N}}(v) \vee \mu_{A^{N}}\left(v_{i}\right)$ where $v_{i}$ are neighbors of $v$ and the busy value of a bipolar fuzzy graph $G$ is defined to be the sum of the busy values of all vertices of $G$, i.e. $D(G)=\sum_{i} D\left(v_{i}\right)$ where $v_{i}$ are vertices of $G$.

Example 1. Let us consider a bipolar fuzzy graph $G=(V, A, B)$ such that $V=\left\{v_{1}, v_{2}, v_{3}, v_{4}\right\}$, and the set of edges $\left\{v_{1} v_{2}, v_{2} v_{3}, v_{1} v_{4}, v_{1} v_{3}, v_{2} v_{4}\right\}$. The membership values of vertices and edges are given by the following table.

\begin{tabular}{|c|ccccc|}
\hline & $v_{1}$ & $v_{2}$ & $v_{3}$ & $v_{4}$ \\
\hline$\mu_{A^{P}}$ & 0.6 & 0.8 & 0.5 & 0.7 \\
\hline$\mu_{A^{N}}$ & -0.3 & -0.4 & -0.6 & -0.5 \\
\hline & $v_{1} v_{2}$ & $v_{2} v_{3}$ & $v_{1} v_{4}$ & $v_{1} v_{3}$ & $v_{2} v_{4}$ \\
\hline$\mu_{B^{P}}$ & 0.5 & 0.1 & 0.4 & 0.3 & 0.7 \\
\hline$\mu_{B^{N}}$ & -0.2 & -0.3 & -0.3 & -0.2 & -0.4 \\
\hline
\end{tabular}

By routine computations, we have $D_{P}\left(v_{1}\right)=1.7, D_{P}\left(v_{2}\right)=1.8, D_{P}\left(v_{3}\right)=1, D_{P}\left(v_{4}\right)=$ 1.3 ,

$D_{N}\left(v_{1}\right)=-0.9, D_{N}\left(v_{2}\right)=-1.1, D_{N}\left(v_{3}\right)=-0.7$, $D_{N}\left(v_{4}\right)=-0.7$.

So, $D\left(v_{1}\right)=(1.7,-0.9), \quad D\left(v_{2}\right)=(1.8,-1.1)$, $D\left(v_{3}\right)=(1,-0.7), D\left(v_{4}\right)=(1.3,-0.7)$.

Definition 14. A vertex $v$ in a bipolar fuzzy graph $G=(V, A, B)$ is said to be a busy vertex if $\mu_{A}^{P}(v) \leqslant$ $\operatorname{deg}_{P}(v)$ and $\mu_{A}^{N}(v) \geqslant \operatorname{deg}_{N}(v)$, otherwise it is called a free vertex.

Definition 15. An edge $u v$ of a bipolar fuzzy graph $G=(V, A, B)$ is said to be an effective edge if $\mu_{B^{P}}(u v)=\mu_{A^{P}}(u) \wedge \mu_{A^{P}}(v)$ and $\mu_{B^{N}}(u v)=\mu_{A^{N}}(u) \vee$ $\mu_{A^{N}}(v)$.

Definition 16. A vertex $v$ of a bipolar fuzzy graph $G=(V, A, B)$ is said to be

(i) a partial free vertex if it is a free vertex in both $G$ and $G^{\mu}$.

(ii) a fully free node if it is a free vertex in $G$, but it is a busy vertex in $G^{\mu}$.

(iii) a partial busy vertex if it is a busy vertex in both $G$ and $G^{\mu}$. 
(iv) a fully busy vertex if it is a busy vertex in $G$, but it is a free vertex in $G^{\mu}$.

Lemma 5. Let $G_{1} \cong G_{2}$ and $h$ be an isomorphism from $G_{1}=\left(V_{1}, A_{1}, B_{1}\right)$ to $G_{2}=\left(V_{2}, A_{2}, B_{2}\right)$. Then $\operatorname{deg}(x)=\operatorname{deg}(h(x))$ for all $x \in V$.

Proof. Since $G_{1} \cong G_{2}$, we have

$\mu_{A_{1}}^{P}\left(x_{1}\right)=\mu_{A_{2}}^{P}\left(h\left(x_{1}\right)\right), \mu_{A_{1}}^{N}\left(x_{1}\right)=\mu_{A_{2}}^{N}\left(h\left(x_{1}\right)\right)$ for all $x_{1} \in V_{1}$.

Hence

$\operatorname{deg}_{P}(x)=\sum_{y \in V_{1}} \mu_{A_{1}}^{P}(y)=\sum_{y \in V_{1}} \mu_{A_{2}}^{P}(h(y))=$ $\operatorname{deg}_{P}(h(x))$

$\operatorname{deg}_{N}(x)=\sum_{y \in V_{1}} \mu_{A_{1}}^{N}(y)=\sum_{y \in V_{1}} \mu_{A_{2}}^{N}(h(y))=$ $\operatorname{deg}_{N}(h(x))$.

Also, we know that $\operatorname{deg}(x)=\left(\operatorname{deg}_{P}(x), \operatorname{deg}_{N}(x)\right)$ for all $x \in V$.

Thus, $\operatorname{deg}(x)=\operatorname{deg}(h(x))$ for all $x \in V$.

Theorem 6. If $G_{1} \cong G_{2}$ and if $v$ is a busy vertex in $G_{1}$, then it is a busy vertex in $G_{2}$ also.

Proof. Let $g: V_{1} \rightarrow V_{2}$ be an isomorphism from $G_{1}$ to $G_{2}$. Then

$\mu_{A_{1}^{P}}(x)=\mu_{A_{2}^{P}}(g(x)), \mu_{A_{1}^{N}}(x)=\mu_{A_{2}^{N}}(g(x))$ for all $x \in V_{1}$ and

$\mu_{B_{1}^{P}}(x y)=\mu_{B_{2}^{P}}(g(x) g(y)), \mu_{B_{1}^{N}}(x y)=\mu_{B_{2}^{N}}(g(x) g(y))$ for all $x y \in E_{1}^{2}$.

Also $g$ preserves the degree of vertices, by Lemma 5, i.e. $\operatorname{deg}_{P}(x)=\operatorname{deg}_{P}(g(x))$, $\operatorname{deg}_{N}(x)=\operatorname{deg}_{N}(g(x))$ for all $x \in V$.

If $x$ is a busy vertex in $G_{1}$, then $\mu_{A_{1}^{P}}(x) \leqslant \operatorname{deg}_{P}(x)$ and $\mu_{A_{1}^{N}}(x) \geqslant \operatorname{deg}_{N}(x)$. Then

$\mu_{A_{2}^{P}}(g(x)) \leqslant \operatorname{deg}_{P}(g(x))$ and $\mu_{A_{2}^{N}}(g(x)) \geqslant$ $\operatorname{deg}_{N}(g(x))$. Hence, $g(x)$ is a busy vertex in $G_{2}$.

Theorem 7. Let a bipolar fuzzy graph $G_{1}$ be weak isomorphism to $G_{2}$. If $u \in V_{1}$ is a busy vertex in $G_{1}$, then its image under a weak isomorphism in $G_{2}$ is also busy.

Proof. Let $g: V_{1} \rightarrow V_{2}$ be a weak isomorphism between $G_{1}$ and $G_{2}$. Then for all $x, y \in V_{1}$

$\mu_{A_{1}}^{P}(x)=\mu_{A_{2}}^{P}(g(x)), \mu_{A_{1}}^{N}(x)=\mu_{A_{2}}^{P}(g(x))$

and $\mu_{B_{1}}^{P}(x y) \leqslant \mu_{B_{2}}^{P}(h(x) h(y)), \quad \mu_{B_{1}}^{N}(x y) \geqslant$ $\mu_{B_{2}}^{N}(g(x) g(y))$
Let $u \in V_{1}$ be a busy vertex. Then $\mu_{A_{1}^{P}}(u) \leqslant \operatorname{deg}_{P}(u)$, $\mu_{A_{1}^{N}}(u) \geqslant \operatorname{deg}_{N}(u)$

From (1) and (3) we have $\mu_{A_{2}^{P}}(g(u))=\mu_{A_{1}^{P}}(u) \leqslant$ $\operatorname{deg}_{P}(u)=\sum_{v \in V_{1}} \mu_{A_{1}^{P}}(v)=\sum_{v \in V_{1}} \mu_{A_{2}^{P}}(g(v))=$ $\operatorname{deg}_{P}(g(u))$. Hence $\mu_{A_{2}^{P}}(g(u)) \leqslant \operatorname{deg}_{P}(g(u))$. Also $\mu_{A_{2}^{N}}(g(u))=\mu_{A_{1}^{N}}(u) \geqslant \operatorname{deg}_{N}(u)=\sum_{v \in V_{1}} \mu_{A_{1}^{N}}(v)$ $=\sum_{v \in V_{1}} \mu_{A_{2}^{N}}(g(v))=\operatorname{deg}_{N}(g(u))$. Therefore, $\mu_{A_{2}^{N}}(g(u)) \geqslant \operatorname{deg}_{N}(g(u))$.

Hence, $g(u)$ is a busy vertex in $G_{2}$.

\section{Categorical properties of bipolar fuzzy graphs}

Many real-world problems can be very effectively described by a graph (e.g. a network), a fuzzy graph, or a bipolar fuzzy graph, but efficient methods to solve such problems often rely on our understanding of the structure of these graphs.

There have been some deeper and untraditional approaches to graph theory (see $[4,5]$ and related references) which are benefit for our understanding of the structure (including limit structure) of graphs and may allure capable pure mathematician in other areas. Research also indicates that category theory may provide a realistic platform on which inter-imitations and inter-inspirations between some fields of mathematics come true [7]. We will show the categorical goodness of bipolar fuzzy graphs.

Definition 17. For a given set $V$, define an equivalence relation $\sim$ on $V \times V-\{(x, x) \mid x \in V\}$ as follows:

$$
\left(x_{1}, y_{1}\right) \sim\left(x_{2}, y_{2}\right) \Leftrightarrow\left\{x_{1}, y_{1}\right\}=\left\{x_{2}, y_{2}\right\} .
$$

The quotient set obtained in this way is denoted as $\widetilde{V^{2}}$, and the equivalent class that contains the element $(x, y)$ is denoted as $[(x, y)], x y$, or $y x$. Note that, $\left(x_{1}, y_{1}\right)$ is an ordered pair and $\left\{x_{1}, y_{1}\right\}$ is an edge.

Theorem 8. The category bipolar fuzzy graph of bipolar fuzzy graphs and homomorphisms between them is isomorphic-closed, complete, and cocomplete.

Proof. Proposition 5.11 of [1] implies that bipolar fuzzy graph is an isomorphic-closed category. Next 
we prove that bipolar fuzzy graph is both complete and co-complete.

Step 1. Bipolar fuzzy graph has equalizers. Let $G_{1}=\left(V_{1}, A_{1}, B_{1}\right)$ (respectively $G_{2}=\left(V_{2}, A_{2}, B_{2}\right)$ be a bipolar fuzzy graph, then $G_{1}, G_{2} \in \operatorname{object(Bipolar}$ fuzzy graph). Assume that $V_{1} \stackrel{f}{\rightrightarrows} V_{2}$ are bipolar fuzzy graph-morphism from $G_{1}$ to $G_{2}$. Let $E=\left\{x \in V_{1} \mid\right.$ $f(x)=g(x)\}$, and $e: E \rightarrow V_{1}$ be an inclusion mapping. We will show that $E \stackrel{e}{\rightarrow} V_{1}$ is an equalizer of $f$ and $g$.

Firstly, let $A=\left.A_{1}\right|_{E}$ and $B=\left.B_{1}\right|_{\mathbb{E}^{2}}$. Then, $G=$ $(V, A, B)$ is a bipolar fuzzy graph on $E$. As $e: E \rightarrow V_{1}$ is an inclusion, we have

$\mu_{A_{1}^{P}}(e(x))=\mu_{A_{1}^{P}}(x)=\mu_{A^{P}}(x), \mu_{A_{1}^{N}}(e(x))=\mu_{A_{1}^{N}}(x)$ $=\mu_{A^{N}}(x), \mu_{B_{1}^{P}}(e(x) e(y))=\mu_{B_{1}^{P}}(x y), \mu_{B_{1}^{N}}(e(x) e(y))$ $=\mu_{B_{1}^{N}}(x y)=\mu_{B^{N}}(x y)$ for each $x y \in E$. Therefore, $e \in$ Morphism (Bipolar fuzzy graph). Obviously, from $G_{E^{\prime}}$ to $G_{1}$ which satisfies $f \circ e^{\prime}=g \circ e^{\prime}$, where $G_{E^{\prime}}=(C, D)$ is a bipolar fuzzy graph of $\left(E^{\prime}, \widetilde{E^{\prime 2}}\right)$. Define $\bar{e}: E^{\prime} \rightarrow E$ by $\bar{e}(x)=e^{\prime}(x) \quad\left(\forall x \in E^{\prime}\right)$. As $f \circ e^{\prime}=g \circ e^{\prime}$, we have $f\left(e^{\prime}(x)\right)=g\left(e^{\prime}(x)\right) \quad(\forall x \in$ $E^{\prime}$ ), thus $\bar{e}$ is well-defined. For each $x \in E^{\prime}$, we have $e \circ \bar{e}(x)=e\left(e^{\prime}(x)\right)=e^{\prime}(x)$, thus $e^{\prime}=e \circ \bar{e}$. As $e^{\prime} \in \operatorname{Morphism}$ (Bipolar fuzzy graph), we have $\mu_{C}^{P}(x) \leqslant \mu_{A_{1}^{P}}\left(e^{\prime}(x)\right)=\mu_{A^{P}}\left(e^{\prime}(x)\right)=\mu_{A^{P}}(\bar{e}(x))$, $\mu_{C}^{N}(x) \geqslant \mu_{A_{1}^{N}}\left(e^{\prime}(x)\right)=\mu_{A^{N}}\left(e^{\prime}(x)\right)=\mu_{A^{N}}(\bar{e}(x))$, $\mu_{D}^{P}(x y) \leqslant \mu_{B_{1}^{P}}\left(e^{\prime}(x) e^{\prime}(y)\right)=\mu_{B^{P}}\left(e^{\prime}(x) e^{\prime}(y)\right)=$ $\mu_{B^{P}}(\bar{e}(x) \bar{e}(y))$,

$\mu_{D}^{N}(x y) \geqslant \mu_{B_{1}^{N}}\left(e^{\prime}(x) e^{\prime}(y)\right)=\mu_{B^{N}}\left(e^{\prime}(x) e^{\prime}(y)\right)=$ $\mu_{B^{N}}(\bar{e}(x) \bar{e}(y))$

for each $x, y \in E^{\prime}$, which implies $\bar{e} \in$ Morphism (Bipolar fuzzy graph). Clearly, such a bipolar fuzzy graph-morphism $\bar{e}$ is unique.

Suppose that $\overline{e^{\prime}}: E^{\prime} \rightarrow E$ is a bipolar fuzzy graphmorphism from $G_{E^{\prime}}$ to $G_{1}$ satisfying $e^{\prime}=e \circ \overline{e^{\prime}}$, then $e \circ \bar{e}=e \circ \overline{e^{\prime}}$, thus we have $\overline{e^{\prime}}(x)=e \circ \overline{e^{\prime}}(x)=$ $e \circ \bar{e}(x)=\bar{e}(x) \quad\left(\forall x \in E^{\prime}\right)$ since $e$ is an inclusion, which implies $\overline{e^{\prime}}=\bar{e}$.

Step 2. Bipolar fuzzy graph has products (this, together with Theorem 12.3 in [6] and step 1, implies that Bipolar fuzzy graph is complete). Assume that $G_{i}=\left(V_{i}, A_{i}, B_{i}\right)$ is a bipolar fuzzy graph of the underlying graphs $G_{i}^{*}=\left(V_{i}, E_{i}\right)(\forall i \in I)$, then $G_{i} \in$ object (Bipolar fuzzy graph) $(\forall i \in I)$.
Let $V=\prod_{i \in I} v_{i}, \quad \pi_{j}: V \rightarrow V_{j}$ be the projection $(\forall j \in I)$, and define bipolar fuzzy sets $A=\left(\mu_{A^{P}}, \mu_{A^{N}}\right)$ and $B=\left(\mu_{B^{P}}, \mu_{B^{N}}\right)$ on $V$ and $\widetilde{V^{2}}$ respectively by $\mu_{A^{P}}\left(\left(x_{i}\right)_{i \in I}\right)=\bigwedge_{i \in I} \mu_{A_{i}^{P}}\left(x_{i}\right)$, $\mu_{A^{N}}\left(\left(x_{i}\right)_{i \in I}\right)=\bigvee_{i \in I} \mu_{A_{i}^{N}}\left(x_{i}\right), \quad \mu_{B^{P}}\left(\left(x_{i}\right)_{i \in I}\left(y_{i}\right)_{i \in I}\right)=$ $\bigwedge_{i \in I} \mu_{B_{i}^{P}}\left(x_{i} y_{i}\right), \quad \mu_{B^{N}}\left(\left(x_{i}\right)_{i \in I}\left(y_{i}\right)_{i \in I}\right)=\bigvee_{i \in I} \mu_{B_{i}^{N}}\left(x_{i} y_{i}\right)$ for any $\left(x_{i}\right)_{i \in I},\left(y_{i}\right)_{i \in I} \in \prod_{i \in I} v_{i}$. Denote $G=(A, B)$, we will show that $\left(G,\left(\pi_{i}\right)_{i \in I}\right)$ is the product of $\left(G_{i}\right)_{i \in I}$. Firstly, as $G_{i}=\left(V_{i}, A_{i}, B_{i}\right)$ is a bipolar fuzzy graph of the underlying graph $G_{i}^{*}=\left(V_{i}, E_{i}\right) \quad(\forall i \in I)$,

$$
\begin{aligned}
& \mu_{B^{P}}\left(\left(x_{i}\right)_{i \in I}\left(y_{i}\right)_{i \in I}\right)=\bigwedge_{i \in I} \mu_{B_{i}^{P}}\left(x_{i} y_{i}\right) \leqslant \bigwedge_{i \in I}\left(\mu_{A_{i}^{P}}\left(x_{i}\right), \mu_{A_{i}^{P}}\left(y_{i}\right)\right) \\
& =\left(\bigwedge_{i \in I} \mu_{A_{i}^{P}}\left(x_{i}\right)\right) \wedge\left(\bigwedge_{i \in I} \mu_{A_{i}^{P}}\left(y_{i}\right)\right)=\mu_{A^{P}}\left(\left(x_{i}\right)_{i \in I}\right) \wedge \\
& \mu_{A^{P}}\left(\left(y_{i}\right)_{i \in I}\right) .
\end{aligned}
$$

Analogously, $\mu_{B^{N}}\left(\left(x_{i}\right)_{i \in I}\left(y_{i}\right)_{i \in I}\right) \geqslant \mu_{A^{N}}\left(\left(x_{i}\right)_{i \in I}\right) \vee$ $\mu_{A^{N}}\left(\left(y_{i}\right)_{i \in I}\right)$.

Therefore, $G \in$ object (Bipolar fuzzy graph).

Secondly, as $\pi_{j}: V \rightarrow V_{j}$ is a projection $(\forall j \in I)$, we have

$$
\begin{aligned}
& \mu_{A^{P}}\left(\left(x_{i}\right)_{i \in I}\right)=\bigwedge_{i \in I} \mu_{A_{i}^{p}}\left(x_{i}\right)=\bigwedge_{i \in I} \mu_{A_{i}^{P}}\left(\pi_{i}\left(\left(x_{i}\right)_{i \in I}\right)\right) \leqslant \\
& \mu_{A_{j}^{p}}\left(\pi_{j}\left(\left(x_{i}\right)_{i \in I}\right)\right), \\
& \mu_{B^{P}}\left(\left(x_{i}\right)_{i \in I}\left(y_{i}\right)_{i \in I}\right)=\bigwedge_{i \in I} \mu_{B_{i}^{P}}\left(x_{i} y_{i}\right)=\bigwedge_{i \in I} \mu_{B_{i}^{P}} \\
& \left(\pi_{i}\left(\left(x_{i}\right)_{i \in I}\right) \pi_{i}\left(\left(y_{i}\right)_{i \in I}\right)\right) \\
& \leqslant \mu_{B_{j}^{P}}\left(\pi_{j}\left(\left(x_{i}\right)_{i \in I}\right) \pi_{j}\left(\left(y_{i}\right)_{i \in I}\right)\right) .
\end{aligned}
$$

Analogously,

$\mu_{A^{N}}\left(\left(x_{i}\right)_{i \in I}\right) \geqslant \mu_{A_{j}^{N}}\left(\pi_{j}\left(\left(x_{i}\right)_{i \in I}\right)\right), \mu_{B^{N}}\left(\left(x_{i}\right)_{i \in I}\left(y_{i}\right)_{i \in I}\right) \geqslant$ $\mu_{B_{j}^{N}}\left(\pi_{j}\left(\left(x_{i}\right)_{i \in I}\right) \pi_{j}\left(\left(y_{i}\right)_{i \in I}\right)\right)$.

Therefore, $\pi_{j} \in$ Morphism (Bipolar fuzzy graph) $(\forall j \in I)$.

Finally, suppose that $H=(V, C, D)$ is a bipolar fuzzy graph of $H^{*}=(X, R)$ and $f_{j}: X \rightarrow V_{j}$ is a bipolar fuzzy graph-morphism from $H$ to $G_{j}(\forall j \in$ $I)$. For each $x \in X$, let $\widehat{f}(x) \in V$ with $\pi_{j}(\widehat{f}(x))$ $=f_{j}(x) \quad(\forall j \in I)$. Then $\widehat{f}: X \rightarrow V$ is a mapping. Since $X \stackrel{f_{j}}{\longrightarrow} V_{j}$ is a bipolar fuzzy graph-morphism $(\forall j \in I), \mu_{C}^{P}(x) \leqslant \bigwedge_{i \in I} \mu_{A_{i}^{P}}\left(f_{i}(x)\right)=\mu_{A^{P}}\left(f_{i}(x)_{i \in I}\right)$ $=\mu_{A^{P}}(\widehat{f}(x)), \quad \mu_{D}^{P}(x y) \leqslant \bigwedge_{i \in I} \mu_{B_{i}^{P}}\left(f_{i}(x) f_{i}(y)\right)=$ 
$\mu_{B^{P}}\left(f_{i}(x)_{i \in I} f_{i}(y)_{i \in I}\right)=\mu_{B^{P}}(\widehat{f}(x) \widehat{f}(y)) . \quad$ Analogously, $\mu_{C}^{N}(x) \geqslant \mu_{A^{N}}(\widehat{f}(x))$ and $\mu_{D}^{N}(x y) \geqslant$ $\mu_{B^{N}}(\widehat{f}(x) \widehat{f}(y))$. Therefore, $f^{n}$ is a bipolar fuzzy graph-morphism with $\pi_{j} \circ \widehat{f}=f_{j} \quad(\forall j \in I)$. Such a bipolar fuzzy graph-morphism $\widehat{f}$ is unique. In fact, if $\bar{f}: X \rightarrow V$ is a bipolar fuzzy graph-morphism satisfying $\pi_{j} \circ \bar{f}=f_{j}(\forall i \in I)$, then $\pi_{j} \circ \bar{f}=\pi_{j} \circ \widehat{f}$. As $\pi_{j}$ is a projection $(\forall j \in I)$, we have $\bar{f}=\widehat{f}$.

Step 3. Bipolar fuzzy graph has co-equalizers. Let $G_{1}=\left(V_{1}, A_{1}, B_{1}\right)$ (respectively, $G_{2}=\left(V_{2}, A_{2}, B_{2}\right)$ be a bipolar fuzzy graph, then $G_{1}, G_{2} \in$ object (Bipolar fuzzy graph). Assume that $V_{1} \stackrel{f}{\rightrightarrows} g V_{2}$ are bipolar fuzzy graph-morphisms from $G_{1}$ to $G_{2}$, $\sim$ is the smallest equivalence relation on $V_{2}$ such that $f(s) \sim$ $g(s)$ for all $s \in V_{1}$. Let $Q=V_{2} / \sim=\left\{[x] \mid x \in V_{2}\right\}$, and $q: V_{2} \rightarrow Q$ be the mapping defined by $q(x)=[x]$ for each $x \in V_{2}$. We will show that $V_{2} \stackrel{q}{\rightarrow} Q$ is a co-equalizer of $f$ and $g$.

Firstly, define bipolar fuzzy sets $A=\left(\mu_{A^{P}}, \mu_{A^{N}}\right)$ and $B=\left(\mu_{B^{P}}, \mu_{B^{N}}\right)$ on $Q$ and $\widetilde{Q^{2}}$ respectively by $\mu_{A^{P}}([x])=\bigvee_{z \in[x]} \mu_{A_{2}^{P}}(z), \quad \mu_{A^{N}}([x])=\bigwedge_{z \in[x]} \mu_{A_{2}^{N}}(z)$, $\mu_{B^{P}}([x][y])=\bigvee_{z \in[x], k \in[y]} \mu_{B_{2}^{P}}(z k), \quad \mu_{B^{N}}([x][y])=$ $\bigwedge_{z \in[x], k \in[y]} \mu_{B_{2}^{N}}(z k)$. Since $G_{1}=\left(V_{1}, A_{1}, B_{1}\right)$ and $G_{2}=\left(V_{2}, A_{2}, B_{2}\right)$ are bipolar fuzzy graphs, we have $\mu_{B^{P}}([x][y]) \leqslant \min \left(\mu_{A^{P}}([x]), \mu_{A^{P}}([y])\right)$, $\mu_{B^{N}}([x][y]) \geqslant \max \left(\mu_{A^{N}}([x]), \mu_{A^{N}}([y])\right)$, which implies $G=(V, A, B)$ is a bipolar fuzzy graph on $Q$. As $\mu_{A_{2}^{P}}(x) \leqslant \bigvee_{z \in[x]} \mu_{A_{2}^{P}}(z)=\mu_{A^{P}}([x])=\mu_{A^{P}}(q(x))$, $\mu_{A_{2}^{N}}(x) \geqslant \bigwedge_{z \in[x]} \mu_{A_{2}^{N}}(z)=\mu_{A^{N}}([x])=\mu_{A^{N}}(q(x))$, $\mu_{B_{2}^{P}}(x y) \leqslant \bigvee_{z \in[x], k \in[y]} \mu_{B_{2}^{P}}(z k)=\mu_{B^{P}}([x][y])=$ $\mu_{B^{P}}(q(x) q(y))$,

$\mu_{B_{2}^{N}}(x y) \geqslant \bigwedge_{z \in[x], k \in[y]} \mu_{B_{2}^{N}}(z k)=\mu_{B^{N}}([x][y])=$ $\mu_{B^{N}}(q(x) q(y))$

for each $x, y \in V_{2}, q \in$ Morphism (Bipolar fuzzy graph). By definition of $\sim, q(f(s))=[f(s)]=$ $[g(s)]=q(g(s))\left(\forall s \in V_{1}\right)$, i.e. $q \circ f=q \circ g$.

Secondly, let $q^{\prime}: V_{2} \rightarrow Q^{\prime}$ be a bipolar fuzzy graph-morphism from $G_{2}$ to $G_{Q^{\prime}}$ which satisfies $q^{\prime} \circ f=q^{\prime} \circ g$, where $G_{Q^{\prime}}=(V, C, D)$ is a bipolar fuzzy graph of $\left(Q^{\prime}, \widetilde{Q^{\prime 2}}\right)$. For each $[x] \in Q$, let $\bar{q}([x])=q^{\prime}(x)$. Then we define, a mapping $\bar{q}: Q \rightarrow$ $Q^{\prime}$. In fact, as $R=\left\{(x, y) \in V_{2}^{2} \mid q^{\prime}(x)=q^{\prime}(y)\right\}$ is an equivalence relation on $V_{2}$ and $q^{\prime} \circ f=q^{\prime} \circ g$, we have $(f(x), g(x)) \in R$, thus $\sim \subseteq R$. For any $x, y \in V_{2}$ satisfying $(x, y) \in \sim$, we have $[x]=[y]$ and $(x, y) \in R$, thus $\bar{q}([x])=q^{\prime}(x)=q^{\prime}(y)=\bar{q}([y])$. As $\bar{q} \circ q(x)$ $=\bar{q}([x])=q^{\prime}(x)\left(\forall x \in V_{2}\right), \bar{q} \circ q=q^{\prime}$.

As $q^{\prime} \in$ Morphism (Bipolar fuzzy graph), we have $\mu_{A^{P}}([x])=\bigvee_{z \in[x]} \mu_{A_{2}^{P}}(z) \leqslant \bigvee_{z \in[x]} \mu_{C}^{P}\left(q^{\prime}(z)\right)$ $=\bigvee_{z \in[x]} \mu_{C}^{P}(\bar{q}([z]))=\mu_{C}^{P}(\bar{q}([z])), \quad \mu_{A^{N}}([x])=$ $\bigwedge_{z \in[x]} \mu_{A_{2}^{N}}(z) \geqslant \bigvee_{z \in[x]} \mu_{C}^{N}\left(q^{\prime}(z)\right)=\bigwedge_{z \in[x]} \mu_{C}^{N}(\bar{q}([z]))$ $=\mu_{C}^{N}(\bar{q}([z])), \quad \mu_{B^{P}}([x][y])=\bigvee_{z \in[x], k \in[y]} \mu_{B_{2}^{P}}(z k) \leqslant$ $\bigvee \mu_{D}^{P}\left(q^{\prime}(z) q^{\prime}(k)\right)$ $z \in[x], k \in[y]$

$\mu_{D}^{P}(\bar{q}([x]) \bar{q}([y]))$,

$$
=\bigvee_{z \in[x], k \in[y]} \mu_{D}^{P}(\bar{q}([z]) \bar{q}([k]))=
$$

$$
\begin{array}{r}
\mu_{B^{N}}([x][y])=\bigwedge_{z \in[x], k \in[y]} \mu_{B_{2}^{N}}(z k) \geqslant \bigwedge_{z \in[x], k \in[y]} \mu_{D}^{N}\left(q^{\prime}(z) q^{\prime}(k)\right) \\
=\bigwedge_{z \in[x], k \in[y]} \mu_{D}^{N}(\bar{q}([z]) \bar{q}([k]))=
\end{array}
$$

$\mu_{D}^{N}(\bar{q}([x]) \bar{q}([y]))$

for each $[x],[y] \in Q$, which implies $\bar{q} \in$ Morphism (Bipolar fuzzy graph). Clearly, such a bipolar fuzzy graph-morphism $\bar{q}$ is unique. Suppose that $\overline{q^{\prime}}: Q \rightarrow Q^{\prime}$ is a bipolar fuzzy graph-morphism from $G$ to $G_{Q^{\prime}}$ satisfying $\overline{q^{\prime}} \circ q=q^{\prime}$, then $\overline{q^{\prime}} \circ q=\bar{q} \circ q$, thus $\overline{q^{\prime}}([x])=\overline{q^{\prime}} \circ q(x)=\bar{q} \circ q(x)=\underline{\bar{q}}([x]) \quad(\forall[x] \in Q)$ by definition of $q$, which implies $\overline{q^{\prime}}=\bar{q}$.

Step 4. Bipolar fuzzy graph has co-products (this, together with Theorem 12.3 in [6] and step 3, implies that bipolar fuzzy graph is complete). Assume that $G_{i}=\left(A_{i}, B_{i}\right)$ is a bipolar fuzzy graph of the underlying graph $G_{i}^{*}=\left(V_{i}, E_{i}\right) \quad(\forall i \in I)$, then $G_{i} \in$ object (Bipolar fuzzy graph) $(\forall i \in I)$.

Let $V=\bigoplus_{i \in I} V_{i}=\bigcup_{i \in I}\left(V_{i} \times\{i\}\right), q_{j}: V_{j} \rightarrow V$ is a mapping satisfying $q_{j}\left(x_{j}\right)=\left(x_{j}, j\right)$ for all $x_{j} \in V_{j}(\forall j \in$ $I)$, and defined bipolar fuzzy sets $A=\left(\mu_{A^{P}}, \mu_{A^{N}}\right)$ and $B=\left(\mu_{B^{P}}, \mu_{B^{N}}\right)$ on $V$ and $\widetilde{V^{2}}$ respectively by $\mu_{A^{P}}\left(x_{i}, i\right)=\mu_{A_{i}^{P}}\left(x_{i}\right), \quad \mu_{A^{N}}\left(x_{i}, i\right)=\mu_{A_{i}^{N}}\left(x_{i}\right)$, $\mu_{B^{P}}\left(\left(x_{i}, i\right)\left(y_{j}, j\right)\right)= \begin{cases}\mu_{B_{i}^{P}}\left(x_{i}, y_{j}\right), & \text { if } i=j, \\ 0 & \text { if } i \neq j,\end{cases}$ 
$\mu_{B^{N}}\left(\left(x_{i}, i\right)\left(y_{j}, j\right)\right)=\left\{\begin{array}{ll}\mu_{B_{i}^{N}}\left(x_{i}, y_{j}\right), & \text { if } i=j, \\ 0 & \text { if } i \neq j,\end{array}\right.$ for each $\left(x_{i}, i\right),\left(y_{j}, j\right) \in V$. Denote $G=(V, A, B)$, we will show that $\left(\left(q_{i}\right)_{i \in I}, G\right)$ is the co-product of $\left(G_{i}\right)_{i \in I}$.

Firstly, as $G_{i}=\left(V_{i}, A_{i}, B_{i}\right)$ is a bipolar fuzzy graph of the underlying graph $G_{i}^{*}=\left(V_{i}, E_{i}\right)(\forall i \in I)$, $G=(V, A, B) \in$ object (Bipolar fuzzy graph).

Secondly, by definition of $q_{j} \quad(\forall j \in I)$, we have $\mu_{A_{j}^{P}}\left(x_{j}\right)=\mu_{A^{P}}\left(x_{j}, j\right)=\mu_{A^{P}}\left(q_{j}\left(x_{j}\right)\right)$ $\mu_{A_{j}^{N}}\left(x_{j}\right)=\mu_{A^{N}}\left(x_{j}, j\right)=\mu_{A^{N}}\left(q_{j}\left(x_{j}\right)\right)$, $\mu_{B_{j}^{P}}\left(x_{j} y_{j}\right)=\mu_{B^{P}}\left(\left(x_{j}, j\right)\left(y_{j}, j\right)\right)=\mu_{B^{P}}\left(q_{j}\left(x_{j}\right) q_{j}\left(y_{j}\right)\right)$, $\mu_{B_{j}^{N}}\left(x_{j} y_{j}\right)=\mu_{B^{N}}\left(\left(x_{j}, j\right)\left(y_{j}, j\right)\right)=\mu_{B^{N}}\left(q_{j}\left(x_{j}\right) q_{j}\left(y_{j}\right)\right)$. Therefore, $q_{j} \in$ Morphism (Bipolar fuzzy graph) $(\forall j \in I)$.

Finally, suppose that $H=(V, C, D)$ is a bipolar fuzzy graph of $H^{*}=(X, R)$ and $g_{j}: V_{j} \rightarrow X$ is a bipolar fuzzy graph-morphism from $G_{j}$ to $H \quad(\forall j \in I)$. Define $\widehat{g}: V \rightarrow X$ by $\widehat{g}\left(x_{i}, i\right)=g_{i}\left(x_{i}\right)$ for each $\left(x_{i}, i\right) \in V$. Obviously, $\widehat{g} \circ q_{j}=g_{j}(\forall j \in I)$. Since $V_{j} \stackrel{g_{j}}{\rightarrow} X$ is a bipolar fuzzy graph-morphism $(\forall j \in I)$, we have $\mu_{A^{P}}\left(x_{j}, j\right)=\mu_{A_{j}^{P}}\left(x_{j}\right) \leqslant$ $\mu_{C}^{P}\left(g_{j}\left(x_{j}\right)\right)=\mu_{C}^{P}\left(\widehat{g}\left(x_{j}, j\right)\right), \quad \mu_{B^{P}}\left(\left(x_{j}, j\right)\left(y_{j}, j\right)\right)=$ $\mu_{B_{j}^{P}}\left(x_{j} y_{j}\right) \leqslant \mu_{D}^{P}\left(\left(g_{j}\left(x_{j}\right) g_{j}\left(y_{j}\right)=\mu_{D}^{P}\left(\widehat{g}\left(x_{j}, j\right) \widehat{g}\left(y_{j}, j\right)\right)\right.\right.$. Analogously, $\mu_{A^{N}}\left(x_{j}, j\right) \geqslant \mu_{C}^{N}\left(\widehat{g}\left(x_{j}, j\right)\right)$, $\mu_{B^{N}}\left(\left(x_{j}, j\right)\left(y_{j}, j\right)\right) \geqslant \mu_{D}^{N}\left(\widehat{g}\left(x_{j}, j\right) \widehat{g}\left(y_{j}, j\right)\right)$. Therefore $\widehat{g}$ is a bipolar fuzzy graph-morphism with $\widehat{g} \circ q_{j}=g_{j} \quad(\forall j \in I)$. Such a bipolar fuzzy graphmorphism $\widehat{g}$ is also unique. In fact, if $\bar{g}: V \rightarrow X$ is a bipolar fuzzy graph-morphism satisfying $\bar{g} \circ q_{j}$ $=g_{j}(\forall j \in I)$, then $\bar{g} \circ q_{j}=\widehat{g} \circ q_{j}$. By definition of $q_{j}(\forall j \in I)$, we have $\bar{g}=\widehat{g}$.

\section{Properties of isomorphism on bipolar fuzzy graphs}

Theorem 9. For any two isomorphic bipolar fuzzy graphs, the order and size are same.

Proof. If $h$ is an isomorphism between the bipolar fuzzy graphs $G_{1}$ and $G_{2}$ with the underlying sets $V_{1}$ and $V_{2}$ respectively, then

$\mu_{A_{1}^{P}}\left(v_{i}\right)=\mu_{A_{2}^{P}}\left(h\left(v_{i}\right)\right)$ and $\mu_{A_{1}^{N}}\left(v_{i}\right)=\mu_{A_{2}^{N}}\left(h\left(v_{i}\right)\right)$, for all $v_{i} \in V$.
$\mu_{B_{1}^{P}}\left(v_{i} v_{j}\right)=\mu_{B_{2}^{P}}\left(h\left(v_{i}\right) h\left(v_{j}\right)\right)$ and $\mu_{B_{1}^{N}}\left(v_{i} v_{j}\right)=$ $\mu_{B_{2}^{N}}\left(h\left(v_{i}\right) h\left(v_{j}\right)\right)$, for all $v_{i}, v_{j} \in V$.

We know that

$O\left(G_{1}\right)=\sum_{v_{i} \in V} \frac{1+\mu_{A_{1}^{P}}\left(v_{i}\right)+\mu_{A_{1}^{N}}\left(v_{i}\right)}{2}$
$=\sum_{v_{i} \in V} \frac{1+\mu_{A_{2}^{P}}\left(h\left(v_{i}\right)\right)+\mu_{A_{2}^{N}}\left(h\left(v_{i}\right)\right)}{2}=O\left(G_{2}\right)$
$S\left(G_{1}\right)=\sum_{v_{i}, v_{j} \in V} \frac{1+\mu_{B_{1}^{P}}\left(v_{i} v_{j}\right)+\mu_{B_{1}^{N}}\left(v_{i} v_{j}\right)}{2}=$
$\sum_{v_{i}, v_{j} \in V} \frac{1+\mu_{B_{2}^{P}}\left(h\left(v_{i}\right) h\left(v_{j}\right)\right)+\mu_{B_{2}^{N}}\left(h\left(v_{i}\right) h\left(v_{j}\right)\right)}{2}=S\left(G_{2}\right)$

Suppose the isomorphism between the bipolar fuzzy graphs $G_{1}=\left(V_{1}, A_{1}, B_{1}\right)$ and $G_{2}=\left(V_{2}, A_{2}, B_{2}\right)$ be weak. Then their order are same. But, if the bipolar fuzzy graphs are of same order need not to be weak isomorphism, which is justified in the following example.

Remark 1. If the bipolar fuzzy graphs be co-weak isomorphism then, their size are same. But, if the bipolar fuzzy graphs are of same size need not to be co-weak isomorphic.

Theorem 10. If $G_{1}$ and $G_{2}$ be isomorphic bipolar fuzzy graphs then, the degrees of their vertices are preserved

Proof. Let $h: V_{1} \rightarrow V_{2}$ be an isomorphism from $G_{1}$ to $G_{2}$. By the definition of isomorphism,

$\mu_{A_{1}^{P}}(x)=\mu_{A_{2}^{P}}(h(x)), \mu_{A_{1}^{N}}(x)=\mu_{A_{2}^{N}}(h(x))$ for all $x \in$ $V_{1}$

$$
\begin{aligned}
& \operatorname{deg}_{P}(x)=\sum_{y \in V_{1}} \mu_{A_{1}^{P}}(y)=\sum_{y \in V_{1}} \mu_{A_{2}^{P}}(h(y))=\operatorname{deg}_{P}(h(x)) \\
& \operatorname{deg}_{N}(x)=\sum_{y \in V_{1}} \mu_{A_{1}^{N}}(y)=\sum_{y \in V_{1}} \mu_{A_{2}^{N}}(h(y))=\operatorname{deg}_{N}(h(x)) .
\end{aligned}
$$

But, the converse of the above theorem is not necessarily true, which is justified in the following example.

Let us consider the two bipolar fuzzy graphs $G_{1}=\left(A_{1}, B_{1}\right)$ and $G_{2}=\left(A_{2}, B_{2}\right)$ which preserve the degree of vertices, but $G_{1}$ and $G_{2}$ are not isomorphic.

By routine computations, we have $\operatorname{deg}\left(v_{1}\right)=\operatorname{deg}\left(u_{1}\right)=(0.5,-1.1)$, $\operatorname{deg}\left(v_{2}\right)=\operatorname{deg}\left(u_{2}\right)=(0.5,-1)$, 
$\operatorname{deg}\left(v_{3}\right)=\operatorname{deg}\left(u_{3}\right)=(0.4,-1.3)$. It is clear that $G_{1}$ and $G_{1}$ are not isomorphic.

\section{Application of related theorems}

A bipolar fuzzy set is an extension of Zadeh's fuzzy set theory whose range of membership degree is $[0,1]$. In a bipolar fuzzy set, the membership degree 0 of an element means that the element is irrelevant to the corresponding property, the membership degree $(0,1]$ of an element indicates that the element somewhat satisfies the property, and the membership degree $[1,0)$ of an element indicates that the element somewhat satisfies the implicit counterproperty. The bipolar fuzzy graph is a generalized structure of a fuzzy graph which gives more precision, flexibility, and compatibility with a system when compared with the fuzzy graphs. The natural extension of the research work on bipolar fuzzy graph is $\mu$-complement and self $\mu$-complement bipolar fuzzy graphs. These results can be applied in database theory, neural networks, geographical information system roughness in graphs, roughness in hypergraphs, soft graphs, and soft hypergraphs.

Fuzzy cognitive maps (FCMs) are used in science, engineering, and the social sciences to represent the causal structure of a body of knowledge (be it empirical knowledge, traditional knowledge, or a personal view); for some examples. An FCM of the type that we shall consider in this paper is described by a set of factors and causal relationships between pairs of factors. A factor can have a direct positive or direct negative impact (or both) on another factor or on itself. In addition, a numerical weight is assigned to each direct impact; these weights are usually taken to be in the interval $[0,1]$. Graphtheoretic tools are used to analyze FCMs. In particular, algorithms for computing a transitive closure of the FCM, from which all, not just direct, impacts together with their weights can be read. Two models can be constructed in the probabilistic model, the absolute value of the weight of an impact is interpreted as the probability that the impact occurs, while in the fuzzy model, it is interpreted as the degree of truth. In both cases, the FCM is represented as a bipolar weighted directed graph; the definition of the transitive closure, however, depends on the model.

Here busy vertices and free vertices in bipolar fuzzy graphs are introduced to improve the solution of the problems. The problem of the probabilistic transitive closure of a bipolar weighted digraph is a bipolar version of the network reliability problem called $\mathrm{s}$, t-connectedness (for all pairs of vertices $s$ and $t$ ). Some of these results mentioned in the paper will help the reduction-recovery algorithm, complete state enumeration, the basic inclusion-exclusion algorithm, and the boolean algebra approach. This adaptation is far from trivial, as care must be taken to generate not only directed paths, but rather all minimal directed walks, and to distinguish between positive and negative minimal directed walks.

\section{Conclusion}

The vertices are the web pages available at the website and a directed edge from page $\mathrm{A}$ to page $\mathrm{B}$ exists if and only if A contains a link to B. The bipolar fuzzy models give more precision, flexibility and compatibility to the system as compared to the classical and fuzzy models. We have introduced some properties of bipolar fuzzy graphs in this paper. The concept of bipolar fuzzy graphs can be applied in various areas of engineering, computer science: database theory, expert systems, neural networks, artificial intelligence, signal processing, robotics, computer networks, and medical diagnosis. In our future work, we will focus on isomorphism properties on highly irregular bipolar fuzzy graphs and define new operations on it. Also, we will study the degree of a vertex in bipolar fuzzy graphs which are obtained from two given bipolar fuzzy graphs $G_{1}$ and $G_{2}$ using the operations cartesian product, composition, tensor and normal product.

\section{References}

1. M. Akram, "Bipolar fuzzy graphs", Information Sciences, 181 5548-5564 (2011).

2. M.Akram and W.A.Dudek, "Regular bipolar fuzzy graphs", Neural Computing and Applications, 21, 197-205 (2012). 
3. K. R. Bhutani, "On Automorphism of fuzzy graphs", Pattern Recognition Lett., 9, 159-162 (1989).

4. R. Diestel, "Locally finite graphs with ends: A topological approach", J, Basic theory, Discrete Mathematics, 311, 1423-1447 (2011).

5. F. Harary, "Graph Theory", third ed., Addison-Wesley, Reading, MA (1972).

6. J. J. Hopfield, "Neurons with graded response have collective computational properties like two-state neurons," Proc. Natl. Acad. Sci., 81, 3088-3092 (1984).

7. P. Komjath, J. A. Larson and N. Sauer, "The quasis order of graphs on an ordinal", Discrete Mathematics, 311, 1451-1460 (2011).

8. J. Lu, S. G. Li, X. F. Yang and W. Q. Fu, "Categorical properties of M-indiscernibility spaces", Theoretical Computer Science, 412, 5902-5908 (2011).

9. J. N. Mordeson and P. S. Nair, "Fuzzy Graphs and Fuzzy Hypergraphs", Physica-Verly, Heidelberg (2000).

10. A. Nagoorgani, and V. T. Chandrasekaran, "Free noodes and busy nodes of a fuzzy graph", East Asian Math. J., 22 (20), 163-170 (2006).

11. A. Rosenfeld, Fuzzy graphs, in: L.A. Zadeh, K.S. Fu, M. Shimura (Eds.), "Fuzzy Sets and Their Applications", Academic Press, New York, 77-95 (1975).

12. H. Rashmanlou, S. Samanta, M. Pal and R. A. Borzooei, "A study on bipolar fuzzy graphs", Journal of Intelligent and Fuzzy Systems, DOI 10.3233/IFS141333 (2014).

13. R. B. Richter, "Graph-like spaces: an introduction", Discrete Mathematics, 311, 1390-1396 (2011).

14. M. S. Sunitha and A. Vijayakumar, "Complement of fuzzy graphs", Indian J. Pure and Appl. Math., 33, 1451-1464 (2002).

15. S. Samanta and M. Pal, "Fuzzy planar graphs", IEEE Transaction on Fuzzy Systems, DOI 10.1109/TFUZZ.2014.2387875 (2015).

16. S. Samanta and M.Pal, "Fuzzy tolerance graphs", International Journal of Latest Trends in Mathematics, 1 (2), 57-67 (2011).

17. S. Samanta and M. Pal, "Fuzzy k-competition graphs and p-competition fuzzy graphs", Fuzzy Inf. Eng., 5 (2), 191-204 (2013).

18. S. Samanta and M. Pal, "Fuzzy threshold graphs", CIIT International Journal of Fuzzy Systems, 3 (12), 360-364 (2011).
19. S. Samanta and M. Pal, "Bipolar fuzzy hypergraphs", International Journal of Fuzzy Logic Systems, 2 (1), 17-28 (2012).

20. S. Samanta and M. Pal, "Irregular bipolar fuzzy graphs", Inernational Journal of Applications of Fuzzy Sets, 2, 91-102 (2012).

21. S. Samanta and M. Pal, "Some more results on bipolar fuzzy sets and bipolar fuzzy intersection graphs", The Journal of Fuzzy Mathematics, 22 (2), 253-262 (2014).

22. S. Samanta, M. Akram and M. Pal, “ $m$-step fuzzy competition graphs", Journal of Applied Mathematics and Computing, 47, 461-472 (2015).

23. S. Samanta, T. Pramanik and M. Pal, "Fuzzy colouring of fuzzy graphs", Afrika Matematika, DOI 10.1007/s13370-015-0317-8 (2015).

24. A. Shannon, K. T. Atanassov, "A first step to a theory of the intuitionistic fuzzy graphs", in: D. Lakov (Ed), Proceeding of FUBEST, Sofia, Sept, 28-30, 5961 (1994).

25. A. Shannon and K. T. Atanassov, "Intuitionistic fuzzy graphs from $\alpha-, \beta-$, and $(\alpha, b)$-Levels", Notes on Intuitionistic Fuzzy Sets 1 (1) 32-35 (1995).

26. A.A. Talebi and H. Rashmanlou, "Isomorphismon on interval-valued fuzzy graphs", Annals of Fuzzy Mathematics and Informatics, 6 (1, 47-58 (2013).

27. D. W. Tank and J. J. Hopfield, "Simple 'neural' optimization networks: An A/D converter, signal decision circuit, and a linear programming circuit," IEEE Trans. on Circuits and Systems, 33, 533-541 (1986).

28. L.A. Zadeh, "Fuzzy Sets", Information and Control, 8, 338-353 (1965).

29. L.A. Zadeh, "Similarity relations and fuzzy orderings", Information Sciences, 3 (2), 177-200 (1971).

30. L.A. Zadeh, "Toward a generalized theory of uncertainty (GTU) an outline", Information Sciences, 172, $1-40$ (2005).

31. W.R. Zhang, "Bipolar fuzzy sets and relations: a computational framework for cognitive modeling and multi agent decision analysis", Proceedings of IEEE Conf., 305-309 (1994).

32. W. R. Zhang, "Bipolar fuzzy sets", Proceedings of FUZZY-IEEE, 835-840,(1998).

33. J. Zhang and X. Yang, "Some properties of fuzzy reasonong in propositional fuzzy logic systems", Information Sciences, 180, 4661-4671 (2010). 